Aus dem Medizinhistorischen Institut der Universität Zürich

(Direktor: Prof.Dr.E.Ackerknecht) und der Medizinischen Universitätspoliklinik Zürich

(Direktor: Prof.Dr.R.Hegglin)

\title{
Zur ersten homologen Tumorübertragung in Zürich durch Arthur Hanau 1889
}

\section{Von H.W.Bucher}

Gewaltig sind die Anstrengungen, die unsere Generation zur Lösung eines der ältesten medizinischen Probleme der Menschheit unternimmt - der Erforschung der Geschwülste. Überblickt man die Entwicklung der letzten fünfzig Jahre, soweit dies einem einzelnen überhaupt möglich ist, so stellt man fest, daß an die Stelle der deskriptiven pathologischen Anatomie die verschiedenen Richtungen der Grundlagenforschung getreten sind, insbesondere die genetische, hormonale, biochemische und virologische. Neben die Beobachtung des vernichtenden Naturexperimentes am Menschen ist die Sammlung einer Vielzahl von Daten und Tatsachen getreten, die als Bausteine in der Zukunft einmal erlauben sollen, das Mosaik der Krebsentstehung zusammenzubauen.

Die Entdeckung zahlreicher Viren in gesunden Organen, Tumoren oder direkt als krebserzeugende Substanzen im Tierversuch hat in verschiedenen modernen Krebstheorien dahin Eingang gefunden, daß ein infektiöses Agens neben anderen Faktoren für die Krebsentstehung von Bedeutung sein könnte. Die moderne Forschung greift damit auf eine Hypothese zurück, die im Verlauf der letzten Jahrhunderte immer wieder im Mittelpunkt lebhafter Diskussionen stand und den Inhalt verschiedener Krebstheorien darstellte. Eng verknüpft mit der Annahme einer infektiösen Ätiologie des Krebses mußte naturgemäß der Versuch sein, auch experimentell Krebsgeschwülste zu übertragen. Daß entsprechende Versuche, die immer wieder angestellt wurden, jahrhundertelang zum Scheitern verurteilt waren, ist der mangelnden Laboratoriumstechnik zuzuschreiben.

Bevor wir auf die erste erfolgreiche Krebsübertragung bei einem Laboratoriumstier durch ARthur Hanau in Zürich eingehen, möchten wir skizzenhaft auf frühere Versuche hinweisen, die etwa im 18. Jahrhundert durch das Aufblühen der pathologischen Anatomie und der Physiologie im modernen Sinn einen brauchbaren Inhalt erhalten haben. 
1774 erschien in Beantwortung einer Preisaufgabe der Akademie von Lyon die Arbeit von B.P.Peyrilhe*, De Cancro (11). Der Autor, der damit gleichzeitig seinen Doktorhut erwarb, erkannte vor allem die einheitliche Natur aller Krebsgeschwülste, in welcher Form und Größe diese auch auftreten mochten. Damit wurden die mangels besserer Kenntnisse noch bis ins 18. Jahrhundert durchgeführten methodischen Trennungen in Scirrhus und Cancer, in blutigen, okkulten, lymphatischen Cancer und wie der deskriptiven Unterscheidungen noch mehr waren hinfällig. Peyrilhe formulierte außerdem die Forderungen, die fortan an die Krebsforschung zu stellen seien, knapp und klar; nämlich die Erforschung 1. der Ursache des Krebsgiftes, 2. der Bestimmung dessen Natur, 3. die Art der Wirkung und 4. die beste Heilmethode. Während die praktische Seite der ganzen Problematik damit gut umrissen war, blieb Peyrilhe in theoretischer Hinsicht allerdings noch stark in den Vorstellungen der cartesianischen Schule befangen. Die überall vorhandene Lymphe stellte nach Descartes die Grundlage jeder Krebsgeschwulst dar**. Die Gründe, die die harmlose Lymphe zu so zügellosem Verhalten zwangen, waren allerdings spekulativ nicht so leicht zu fassen. Peyrilhe zog immerhin den naheliegenden Schluß, daß durch die Annahme eines Krebsvirus das Problem einer Lösung nähergebracht werden könnte. Konsequenterweise versuchte er daher auch durch Tierexperimente den konkreten Nachweis der Krebsübertragbarkeit zu erbringen. Seinen Hundeexperimenten war durch widrige äußere Umstände kein Erfolg beschieden. Mit der Preisarbeit Peyrilhes, die unter den Zeitgenossen große Beachtung fand, hatten die positiven Ansätze zur Lösung des Krebsproblems vorläufig wieder ein Ende gefunden. Das Endresultat, zu dem er gekommen war: "Cette maladie est aussi difficile à définir qu'à guérir», hat leider seinen tragischen Sinn bis heute nicht verloren.

Die Gründe, die eine weitere Forschung im Sinne Peyrilhes nicht aufkommen ließen, sind leicht zu übersehen. Um die Jahrhundertwende öffnete Віснат mit seiner analytischen Gewebelehre die Tore für feingewebliche Studien, die er selbst auch bei Tumoren systematisch durchzuführen begann. Er begründete damit, unterstzützt von seinen Schülern, eine Solidarpathologie, die allerdings noch fast drei Jahrzehnte einen schweren Stand gegen die spekulative humoralpathologische Schule Broussais haben sollte. Broussais vertrat mit dem ganzen Gewicht des wortgewaltigen Klinikers

* 1735-1804.

** Zitiert nach J. WoLfF (13). 
die Auffassung, daß die Ursache des Krebses in einer chronischen Entzündung zu suchen sei, und lehnte eine Krebskrankheit sui generis rundweg ab, wie sie von Lennec, BaYle und CaYol schon 1812 im Dictionnaire des sciences médicales klar dargestellt worden war. In der Folge bekam die histologische Forschung durch die verbesserten Färbetechniken und Mikroskope einen so starken Auftrieb, daß besonders die Pathologen dem Kontagiositätsproblem und der Frage von experimentellen Übertragungen von Tumoren keine Aufmerksamkeit schenken konnten. Die Entdeckung der Bakterien gab zwar einzelnen Theoretikern Anlaß, auch für den Krebs eine bazilläre Ursache anzunehmen. Vırchow, mit dem Problem konfrontiert, nahm lange Zeit eine schwankende, letzten Endes aber doch eine negative Stellung ein. Als ätiologisches Moment wäre für ihn ein Virus oder Bazillus durchaus möglich gewesen, der, ins Bindegewebe eingedrungen, über eine formative Entzündung die Bildung von heterologen Epithelmassen auslösen könnte. Für eine endgültige bazilläre Theorie schienen ihm aber die experimentellen Grundlagen noch zu fehlen (12).

Trotzdem wurden während des ganzen 19. Jahrhunderts besonders von klinischer Seite immer wieder Versuche unternommen, den Krebs von Tier auf Tier oder von Menschen auf Tiere zu übertragen. Auf die durchaus negativ verlaufenen Versuche von Langenbeck, Follin, Lebert, Velpeau und Billroth sei hier nur am Rande verwiesen. Eine genaue Beschreibung der Versuchsanordnungen und Resultate gibt Jаков WolfF (13).

Im Jahre 1889 veröffentlichte der kaum dreißigjährige Privatdozent Arthur Hanau* aus dem Zürcher Pathologischen Institut, das derzeit unter der Leitung von KLEBS stand, in einer kritischen und durch histologische Untersuchungen wohlfundierten Arbeit die erfolgreiche Übertragung eines Pflasterzellkarzinoms aus der Vulva einer Ratte auf ein anderes

* Arthur Hanau, 1858 in Frankfurt geboren, stammte aus einer wohlhabenden jüdischen Geschäftsfamilie. Seine Medizinstudien absolvierte er in Marburg, Bonn, Leipzig, wobei wir unter seinen Lehrern Namen wie Cohnheim, Ribbert, Strümpeld, Thiersch, Erb, CredÉ und Ludwig finden. Seine eigentliche Tätigkeit als Pathologe begann um 1882 bei EmIL Ponfick in Breslau. Obschon er bald die beschränkten Möglichkeiten für eine eigene fruchtbare experimentelle Tätigkeit erkannte, harrte er etwa drei Jahre bei Ponfick aus, dem offenbar gesellschaftliche Verpflichtungen wichtiger waren als eine zeitgemäße Förderung seines Faches. Nach Zürich in das Institut von EdwIN KLEBS übersiedelt, habilitierte er sich für das Gebiet der pathologischen Anatomie und hielt während mehrerer Semester zusammen mit Lubarsch die meisten Vorlesungen in diesem Fach, das von Klebs stark vernachlässigt wurde. Mehrere vorzügliche Arbeiten aus dieser Zeit lassen erkennen, wie fruchtbar sich das unabhängige experimentelle Arbeiten 
Tier der gleichen Spezies (1). Kurz vorher hatte er die Versuchsresultate in einer kurzen Demonstration am 18. Kongreß der Deutschen Gesellschaft für Chirurgie in Berlin vorgetragen (2). Die Übertragung geschah durch die Implantation eines frisch gewonnenen Krebspartikels auf ein männliches Tier, und zwar ins Scrotum, das bei der Ratte eine direkte Kommunikation mit der Bauchhöhle besitzt. Bei der Autopsie des zwei Wochen später spontan eingegangenen Tieres wurde eine massive Ausbreitung des Tumors in die Bauchhöhle beobachtet. Die histologische Untersuchung zeigte ein völliges Übereinstimmen im ganzen Bau und im Grade der Differenzierung mit dem Primärtumor.

Diesem experimentellen Erfolg kommt in der Geschichte der Krebsforschung eine gewisse Bedeutung zu. Es ist aber nicht, wie es vorerst scheinen möchte, das Gelingen eines besonders schwierigen Versuches oder die Originalität der Versuchsanordnung, in welcher der Wert der Arbeit begründet ist. Wie wir oben schon festgestellt haben, wurden Experimente ähnlicher Art schon früher von verschiedenen Klinikern angestellt. Hanau selbst verweist auch auf zwei kurze Mitteilungen von Novinsky (14) und WeHr 15, 16), die nach seiner Auffassung eindeutig positive Resultate zeitigten. Da es sich dabei aber nur um kurze kasuistische Mitteilungen handelt, die uns keinen Aufschluß über die endgültigen Schlußfolgerungen der Autoren geben, sind sie für die historische Auswertung von beschränktem Wert.

Die Bedeutung der Hanauschen Publikation sehen wir darin, daß er seine in ihrer Art völlig neuen Versuchsresultate mit dem damaligen Stand der Tumorpathologie konfrontierte und eine Interpretation zu geben versuchte. Auf Grund der präzisen Beschreibung kann nicht daran gezweifelt werden, daß es sich um eine echte Tumorübertragung mit selbständigem Weiterwachsen und Metastasierung und nicht um ein bloßes Überleben von Tumormaterial gehandelt hat. Hanau hatte damit die lange bezweifelte Möglichkeit der Verimpfbarkeit der Karzinome auf Tiere gleicher Art erbracht. Zwar hatte PAUL BERT 1863 schon das Überleben von transpla-

auswirkte. Ernsthafte Auseinandersetzungen mit Klebs veranlaßten ihn, Zürich zu verlassen und als Privatgelehrter am Kantonsspital St.Gallen seine pathologischen Forschungen weiterzuführen. Zeitweise unterhielt er in Zürich noch ein Privatlaboratorium, in dem er Kurse für Studenten abhielt. Mit vierzig Jahren erkrankte Hanau an einem Darmkrebs, dessen unaufhaltsames Voranschreiten er nicht mehr mitansehen wollte. Sein selbstgewählter Tod erfolgte am 2.August 1900 in Konstanz, wo er auch begraben liegt. 
nierten Rattenschwänzen beobachtet (17) und REvERDIN 1869 über das Überleben von Hauttransplantaten berichtet, die sich dem neuen Organismus völlig anpaßten (18). Bei den Tieren Hanaus lag aber ein richtiges expansives Wachstum mit Metastasierung vor, dessen Erklärung nicht ohne weiteres aus den Vorstellungen der allgemeinen Pathologie geschöpft werden konnten.

Als erstes lehnt es Hanau ab, aus seinen Versuchen auf eine infektiöse Ursache der Krebsbildung irgendwelche Rückschlüsse zu ziehen. Als Histopathologe sah er in der Tumorzelle den eigentlichen Träger der gesteigerten Wachstumspotenz. Dies war mindestens in der zweiten Hälfte des 19. Jahrhunderts die Ansicht der meisten zünftigen Pathologen, unter ihnen Cohnheim, Thiersch u. a., die auf Hanau einen nicht unbedeutenden Einfluß ausgeübt haben. Neben dieser mehr konformistischen Ansicht gab es immerhin einzelne namhafte Pathologen, wie z.B. E. Kaufmann, die aus der Entdeckung der Bakterien spekulativ Rückschlüsse auf die Krebsfrage zu ziehen wagten. Die Äußerung Kaufmanns: «die Carcinomfrage würde nun auch (wie die Tuberkulosefrage) durch die Auffindung eines spezifischen Mikroorganismus am einfachsten gelöst werden », wurde denn auch von Hanau eindeutig abgelehnt (1). Gerade für die Tuberkulose war die spezifische Wirkung der Tuberkelbazillen auf die Zellen genau studiert worden. Hanau selbst hatte sich intensiv mit der Histologie der Tuberkulose in seiner Habilitationsschrift beschäftigt (3) und hatte ebenfalls die direkte nekrotisierende Wirkung der Tuberkelbakterien auf die Zellen, die schließlich zum eigentlichen Tuberkelknötchen sich entwickeln, beobachten können, wie dies schon einige Jahre vorher WEIGERT an den Langhansschen Riesenzellen beschrieben hatte (19). Während hier wie bei anderen Infektionen die Wirkung der Mikroorganismen Nekrose und Entzündung waren, zeigten die Krebsgeschwülste eine völlig andere Histogenese. Hanau folgerte deshalb: "Die Annahme, daß ein Mikroorganismus beispielsweise ein Endothel in ein verhornendes Plattenepithel umwandeln könne, wäre auf Grund unserer heutigen Kenntnisse ebensowenig zu rechtfertigen wie die Hypothese, daß ein symbiotisch lebender (Mikroorganismus) der Epithelzelle eine höhere Proliferationsfähigkeit und die Fähigkeit zu metastasieren verliehe und dabei überdies noch unfähig wäre, auf die Epithelien secundär befallener Organe oder sogar secundär betroffener Stellen des gleichen Organes überzugehen.»

Hanau lehnte somit auf Grund morphologischer Analogien eine infektiöse Krebsgenese ab. Interessanterweise hatte die Vorstellung von einer 
eigentlichen Ansteckungsfähigkeit des Krebses bei Klinikern nie ganz an Boden verloren. Im 17. und 18. Jahrhundert hielt man allgemein den Krebs für ebenso ansteckend wie die Phthise, und diese Ansicht hat sich durch das ganze 19. Jahrhundert ihre überzeugten Anhänger bewahrt. Zu dieser Meinung war man vor allem gestützt auf zahllose kasuistische Beobachtungen gekommen, die in die Literatur u. a. als sogenannte "cancer à deux» eingegangen sind. Noch vor sechzig Jahren schrieb z.B. der französische Kliniker O.GueILlot : «Une maison où un cancéreux a succombé est contaminée et doit être desinfectée» $(21,22)$.

Die Arbeit Hanaus fand in der zeitgenössischen Lehrbuchliteratur einen gewissen Widerhall, jedoch keine eigentliche Bestätigung. Auch die eigenen Experimente wurden nicht lange weitergeführt. LubaRsch weiß viele Jahre später in seiner Autobiographie zu berichten, daß KLEBs, dessen Neid erregt worden war, auf administrativem Weg die weitere Fütterung der Ratten unterbunden habe (23). Edwin Klebs, dessen Verdienste als genialer Forscher auf dem Gebiet der Pathologie und Bakteriologie unbestritten sind, hatte es verstanden, sich in Zürich zahlreiche Feinde zu schaffen. Insbesondere hatte er sich, wie RöTHLIN nachgewiesen hat, 1885 in Fragen der Wasserversorgung der Stadt Zürich sehr stark exponiert (24). Hinzu $\mathrm{kam}$, daß er während vieler Jahre den Unterricht völlig vernachlässigte. Da nach dem Abgang von Lubarsch und etwas später von Hanau die Pathologie in Zürich nicht mehr geordnet vorgetragen wurde, mußte Klebs 1892, besonders wegen des starken Druckes von seiten der Studenten, sein Ordinariat niederlegen.

LEIBBRAND hat kürzlich die äußeren Lebensdaten Hanaus an Hand seines Nachlasses zusammengestellt und dabei auf dessen wechselnden Charakter hingewiesen, der von der sensiblen Hypochondrie bis zur leidenschaftlichen Agressivität wechseln konnte (25). Wie wir aus einer Sammlung seiner Briefe an seinen Zürcher Freund Konstantin v. Monakow entnehmen können, scheinen sich eine deutlich depressive Verstimmung mit gewissen hypochondrischen Zügen erst in den Jahren nach dem unfreiwilligen Abgang von Klebs eingestellt zu haben*. Zweifellos hatte Hanau mit einer Berufung nach Zürich gerechnet, die ihm wiederum ein größeres Wirkungsfeld eröffnet hätte. Seine Enttäuschung ging langsam in einen richtigen Ekel vor dem akademischen Betrieb über. Die letzten Jahre bis zu seinem

* Wir verdanken Herrn Dr. med. K. HarmmanN-v. Monakow, Zürich, die Überlassung des Briefwechsels. 
vorzeitigen Tod brachten ihm einzig noch die Befriedigung der wissenschaftlichen Arbeit. Im Alter von 42 Jahren setzte Hanau seinem Leben selbst ein Ende. Von atheistischer Weltanschauung, sah er sich durch ein heimtückisches Krebsleiden in seiner körperlichen Integrität beeinträchtigt und wollte sich selbst das Beobachten seines körperlichen Zerfalls ersparen. Dieses Ende entbehrt nicht der Tragik. Für die medizinische Forschung bedeutete Hanaus Tod zweifellos einen Verlust, waren doch auch damals schon originelle Forscherpersönlichkeiten eher eine Seltenheit. Neben der besprochenen Arbeit über Krebs und seines Habilitationsschrift über die Lungentuberkulose hatte er noch über Knochenveränderungen in der Gravidität (4), über die Entstehung und Zusammensetzung der Thromben $(5,6)$, über den damaligen Stand der Lehre von der Heilung und der Immunität (7), über die Osteomalazie (8), über die Schilddrüse bei Cretinismus (9) sowie über experimentelle Arbeiten über die Physiologie der Darmsekretion (10) vielbeachtete Arbeiten veröffentlicht. Sie alle zeugen von einem kritischen, ganz der experimentellen Forschung zugetanen Geist. Seine Versuche zum Krebsproblem verdienen jedenfalls einen definitiven Platz in der Wissenschaftsgeschichte im allgemeinen und der Zürcher Fakultät im besondern.

1 A. Hanau, Erfolgreiche experimentelle Übertragung von Carcinom, Fortschr. Med. 7 (1889) 321.

2 - Experimentelle Übertragung von Carcinom von Ratte auf Ratte (Vortrag mit Demonstration, 18. Congreß der Deutschen Gesellschaft für Chirurgie, Berlin 1889), Arch.klin.Chir.39(1889) 678.

3 - Habilitationsschrift: Beiträge zur Pathologie der Lungenkrankheiten. I. Über Lokalisation und weitere Verbreitung der Tuberkulose in der Lunge. II. Zur Histologie der croupösen und indurierten Pneumonie. Z. klin. Med. 12 (1887) 1, gedruckt bei L. Schumacher, Berlin 1886.

4 - Über Knochenveränderungen in der Schwangerschaft und über die Bedeutung des puerperalen Osteophyts, Fortschr. Med.10 (1892) 237.

5 - Zur Entstehung und Zusammensetzung der Thromben, Fortschr. Med.4 (1886) 385.

6 - Nochmals zur Entstehung und Zusammensetzung der Thromben, Fortschr.Med.5 (1887) 65 .

7 - Einige Bemerkungen über den heutigen Stand der Lehre von der Heilung und der Immunität, Fortschr.Med.6 (1888) 849.

8 - Ein Vorschlag zur Lösung der Streitfrage, ob der kalklose Knochen bei der Osteomalazie durch Knochenberaubung oder durch Ausbleiben der Verkalkung entsteht, Fortschr.Med.18 (1900) 81. 
9 - Demonstration mikroskopischer Präparate von Atrophie der Schilddrüse bei Cretinismus, mit Bemerkungen über das Verhältnis von Cretinismus zu Myxoedem, Verhandlungen des X. Internationalen Medizinischen Kongresses, Bd. 2, 1891, Sektion III, S. 128.

10 - Experimentelle Untersuchungen über die Physiologie der Darmsekretion, Z. Biol. $22(1886) 195$.

11 B. Peyrille, Diss. De Cancro ..., Paris 1774.

12 R.Virchow, Die Krankhaften Geschwülste. Vorlesungen gehalten 1862-63 in Berlin. 5.Vorlesung: Die Pathogenie der neoplastischen Geschwülste. Berlin 1863.

13 JAK. WolfF, Die Lehre von der Krebskrankheit, Band I, Jena 1909.

14. M.Nowinsky, Zur Frage über die Impfung der krebsigen Geschwülste, Centralblatt für die Medizinischen Wissenschaften 14 (1876) 790.

15 WeHr, Demonstration der durch Impfung von Hund auf Hund erzeugten Carcinomknötchen, Centralblatt für Chirurgie 24 (1888) 8.

16 - Weitere Mitteilungen über die positiven Ergebnisse der Carcinom-Überimpfung von Hund auf Hund, Arch.klin. Chir. 39 (1889) 226.

17 P.Bert, zitiert nach HaNAU (1).

18 J.L.Reverdin, Greffe épidermique, Bull. Soc. Imp. Chir. 10 (1870) 511.

19 C. Weigert, Zur Theorie der tuberkulösen Riesenzellen, Dtsch. med. Wschr. 35 (1885) 599.

20 - Über Metschnikoff's Theorie der tuberkulösen Riesenzellen, Fortschr. Med. 6 (1888) 809.

21 O. GueIllot, La contagion du cancer, Gazette des hôpitaux 1892.

22 - La question du cancer, Union Med. du Nord-Est 1891.

23 O.Lubarsch, Ein bewegtes Gelehrtenleben, Berlin 1931.

24 O.M.RöтнLIN, Edwin Klebs (1834-1913), ein früher Vorkämpfer der Bakteriologie, und seine Irrfahrten.

25 W.Leibbrand, Arthur Hanau, Med. Klinik 58 (1963) 1280. 